\title{
BIOESTRATIGRAFIA (FORAMINIFERIDA) DA SONDAGEM 1-SCS-3B, PLATAFORMA DE FLORIANÓPOLIS, BACIA DE PELOTAS, BRASIL
}

\author{
GEISE DE SANTANADOS ANJOS \\ UFRGS, Programa de Pós-graduação em Geociências, Cx. P. 15.001, 91501-970, Porto Alegre, RS, Brasil. \\ geise.anjos@ufrgs.br \\ ANA LUISA CARREÑO \\ Universidad Nacional Autónoma de México, Instituto de Geología, Delegación de Coyoacán, 04510 \\ D.F. México, México.anacar@servidor.unam.mx
}

\begin{abstract}
RESUMO - A microfauna de foraminíferos planctônicos recuperada da sondagem 1-SCS-3B, Plataforma de Florianópolis, Santa Catarina, está representada por 60 espécies e subespécies pertencentes a 13 gêneros, cuja distribuição estratigráfica indica idade neogênica para os sedimentos estudados. A análise desta assembléia permitiu o reconhecimento de oito biozonas de intervalo, em ordem estratigráfica: Catapsydrax dissimilis, $C$. stainforthi, Globorotalia fohsi fohsi, Globigerinoides ruber, Globorotalia mayeri, G. acostaensis/G. menardii, G. margaritae evoluta e Globigerinoides trilobus fistulosus. Foram identificados quatro hiatos importantes situados no limite Oligoceno-Mioceno, Mioceno inferior, limite Mioceno inferior-Mioceno médio e no limite Mioceno-Plioceno. Correlações com zoneamentos prévios para a área são aqui discutidas.
\end{abstract}

Palavras-chave: Foraminiferida, bioestratigrafia, Neogeno, bacia de Pelotas.

\begin{abstract}
BIOSTRATIGRAPHY (FORAMINIFERIDA) OF THE 1-SCS-3B DRILL-HOLE, PLATAFORMA DE FLORIANÓPOLIS, PELOTAS BASIN, BRAZIL. Planktonic foraminifers recovered from 1-SCS-3B drill hole, Florianópolis Platform, State of Santa Catarina, Brazil, represent 60 species and subspecies belonging to 13 genera, whose stratigraphic range indicates a Neogene age for the sediments herein studied. The analysis of this assemblage allowed the recognition of eight zones, in stratigraphic order: Catapsydrax dissimilis, C. stainforthi, Globorotalia fohsi fohsi, Globigerinoides ruber, Globorotalia mayeri, G. acostaensis/ G. menardii, G. margaritae evoluta and Globigerinoides trilobus fistulosus. Four important hiatuses were observed in the studied sedimentary sequence, whose are situated at Oligocene-Miocene boundary, Early Miocene, Early Miocene-Middle Miocene and Miocene-Pliocene boundary. Correlations with previous zonations for the area are discussed.
\end{abstract}

Key words: Foraminiferida, biostratigraphy, Neogene, Pelotas basin.

\section{INTRODUÇÃO}

A Bacia de Pelotas tem sido alvo de diversos estudos bioestratigráficos, paleoecológicos e de cunho taxonômico baseados em variados grupos de microfósseis. Estudos sobre a microfauna de foraminíferos do Neocenozóico foram apresentados por Closs (1966a, b, 1967, 1970), Closs \& Madeira (1968), Fernandes (1975), Thiesen (1975), Madeira-Falcetta et al. (1980), Thiesen \& Madeira-Falcetta (1984), Madeira-Falcetta \& Thiesen (1988), Koutsoukos (1982) e Boltovskoy et al. (1983). Bertels \& MadeiraFalcetta (1977) apresentaram uma síntese do conhecimento a cerca das assembléias de foraminíferos planctônicos do Neogeno das bacias do Atlântico Sul, incluindo a Bacia de Pelotas. Na porção uruguaia da Bacia de Pelotas, Sprechmann (1978) analisou assembléias de foraminíferos bentônicos e moluscos do Neogeno e do Quaternário, enquanto Kotzian \& Eilert (1985) estudaram os ostracodes mio-pleistocênicos.

Diversos trabalhos baseados no estudo de ostracodes foram realizados na seção neogênica emersa da bacia, dentre os quais destacam-se os de Sanguinetti (1979, 1980), Ornellas (1981), Sanguinetti et al. (1991, 1992), Carreño et al. (1997, 1999) e Carmo \& Sanguinetti (1999). Outros grupos de microfósseis do Neocenozóico também foram estudados: Daemon (1969) apresentou dados taxonômicos e bioestratigráficos baseados em palinomorfos; Gomide (1989) apresentou uma contribuição à biocronoestratigrafia com base no estudo de assembléias de nanofósseis calcários.

Apesar dos trabalhos acima citados, a bioestratigrafia do Neogeno da bacia de Pelotas ainda não está suficientemente estudada, visto que a maioria dos autores se deteve na aná- 


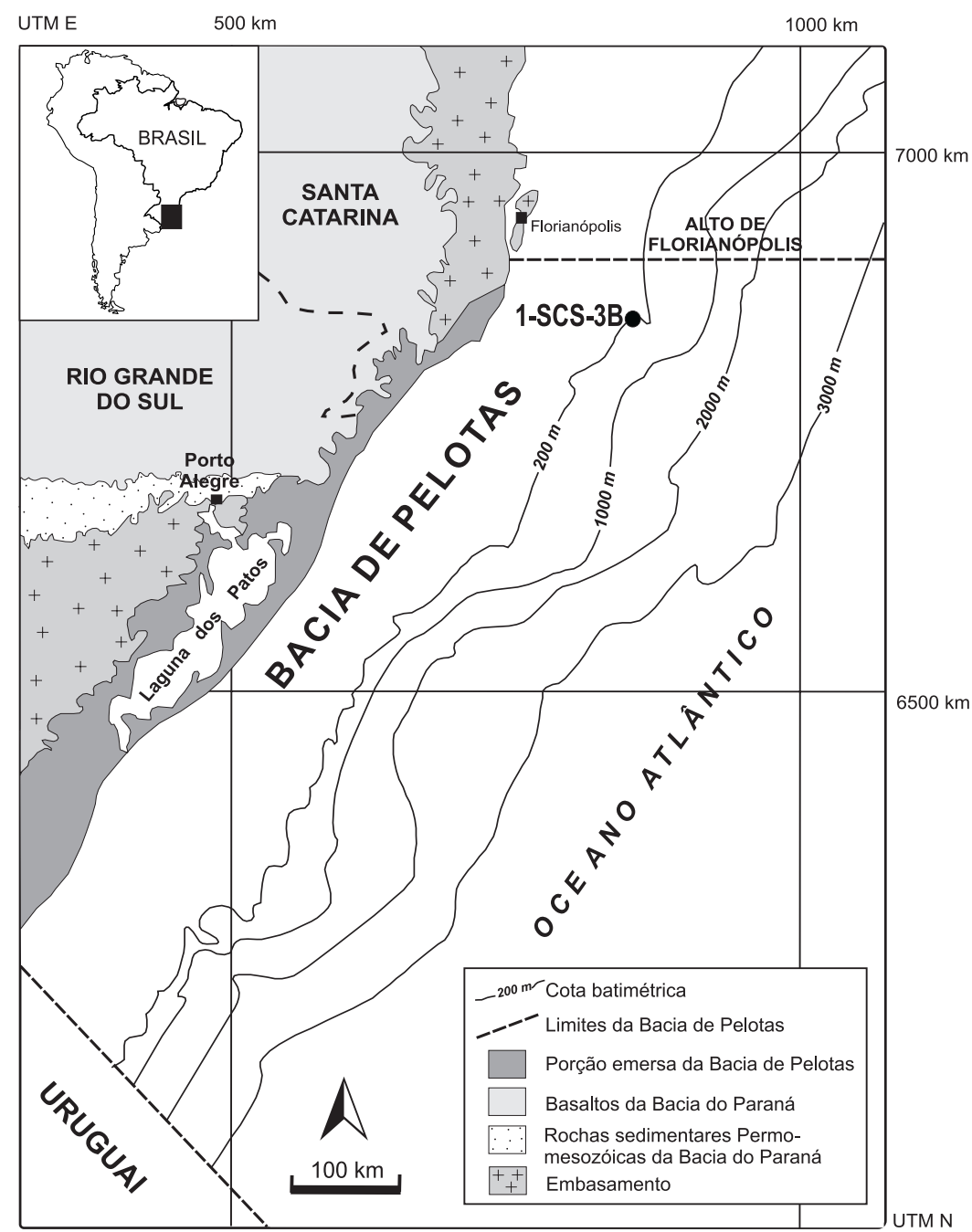

Figura 1. Mapa de localização da bacia de Pelotas e da sondagem estudada. Figure 1. Location map of the Pelotas basin and the study drill hole.

lise das assembléias de microfósseis recuperadas de sondagens onshore efetuadas pela Petrobrás. Dos trabalhos anteriores, apenas Koutsoukos (1982) e Gomide (1989) estudaram material proveniente de poços offshore perfurados na Plataforma de Florianópolis, inclusive o poço 1-SCS-3B.

No presente trabalho apresenta-se uma proposta bioestratigráfica para o Neogeno da Plataforma de Florianópolis, porção setentrional da Bacia de Pelotas. Foi analisada a microfauna de foraminíferos planctônicos da sondagem offshore 1-SCS-3B, da Petrobrás, e procedeu-se à correlação dos resultados obtidos neste poço com outras propostas de biozoneamento para a área.

\section{CONTEXTOGEOLÓGICO}

A Bacia de Pelotas compreende o trecho da margem continental sul-brasileira localizada entre o Alto de Florianópolis e a fronteira com o Uruguai (Figura 1). A porção uruguaia da Bacia de Pelotas é conhecida como Cuenca del Leste e se estende até o alto do embasamento de La Coronilla.

A bacia tem direção geral NE-SW e ocupa uma área de 210.000 $\mathrm{km}^{2}$ até a isóbata de $2.000 \mathrm{~m}$, sendo que destes, $40.000 \mathrm{~km}^{2}$ estão emersos. Do ponto de vista tectônico, a Bacia de Pelotas se constitui na precursora das bacias brasileiras de margem passiva (Gonçalves et al., 1979; Asmus, 1983).

Gonçalves et al. (1979) individualizaram a porção setentrional da Bacia de Pelotas, denominando-a Plataforma de Florianópolis. Esta teria se desenvolvido sobre uma antiga zona de fraqueza, provavelmente uma ramificação da Zona de Fratura de Rio Grande (Gamboa \& Rabinovitz, 1981).

As rochas que constituem o preenchimento sedimentar da Bacia de Pelotas e da Plataforma de Florianópolis sobrepõem-se diretamente ao embasamento ou, localmente, a rochas da Bacia do Paraná. O intervalo aqui estudado está inserido no contexto litoestratigráfico das formações Cidreira e Imbé. A primeira corresponde a clásticos finos a grossos com idades entre o Turoniano e o Holoceno, provavelmente associados a ambientes transicionais. A Formação Imbé é constituída por depósitos pelíticos com raras intercalações de areias turbidíticas, depositados em ambiente marinho profundo, com idades entre o Turoniano e o Recente (Dias et al., 1994). A sucessão sedimentar neogênica tem caráter regressivo, tendo sido dividida em cinco seqüências deposicionais limitadas por superfícies erosivas (Koutsoukos, 1982, Fontana, 1996).

Fontana (1996), utilizando estratigrafia de seqüências, propôs cartas cronoestratigráficas distintas para a Bacia de Pelotas e a Plataforma de Florianópolis. Entretanto, a arquitetura estratigráfica para as seções mio-pliocênicas nas duas cartas apresenta forte similaridade, de modo que consideramos neste trabalho as mesmas unidades para ambas as províncias.

\section{MATERIALE MÉTODOS}

Foram analisadas 34 amostras de calha provenientes do poço 1-SCS-3B, perfurado pela Petrobrás na porção offshore da Bacia de Pelotas (28²9'34,18' S, 47²9'10,8' W). A perfuração alcançou $4.738 \mathrm{~m}$ de profundidade e as amostras estudadas foram coletadas no trecho compreendido entre $1.320 \mathrm{e}$ 360 metros de profundidade, com intervalo amostral de $15 \mathrm{~m}$.

De cada amostra foram processados $40 \mathrm{~g}$ de sedimentos. As amostras foram adicionadas de peróxido de hidrogênio 34 volumes até a sua completa desagregação, sendo em seguida lavadas em peneiras de malha $0,250 \mathrm{~mm}, 0,177 \mathrm{~mm}$ e $0,062 \mathrm{~mm}$ e secas em estufa a $60^{\circ} \mathrm{C}$. Foram examinadas as duas primeiras frações, com recuperação da microfauna total de foraminíferos.

A classificação sistemática adotada neste trabalho é aquela apresentada por Loeblich \& Tappan (1988) até o nível genérico. Em nível específico e subespecífico, a classificação 
foi baseada em Bolli \& Saunders (1985) com o auxílio de bibliografia complementar (Kennet \& Srinivasan ,1983; Stainforth et al., 1975; Ellis \& Messina, 1995; Blow, 1969; Bolli \& Saunders, 1981, 1982; Bolli \& Premoli Silva, 1973).

O material analisado está tombado na coleção de foraminíferos do Museu da Universidade Federal do Rio Grande do Sul, sob a denominação Foraminiferida (Bacia de Pelotas). Os espécimes figurados receberam a numeração MP-F-3085 a MP-F-3096.

No estabelecimento do arcabouço bioestratigráfico foi adotado o esquema zonal de Bolli \& Saunders (1985). As idade dos limites das zonas correspondem àquelas dos data de extinção dos táxons estabelecidos por Berggren et al. (1995).

Exemplares representativos das espécies-índice de cada biozona foram fotografados em microscópio eletrônico de varredura modelo JEOL JSM-5800, do Centro de Microscopia Eletrônica da Universidade Federal do Rio Grande do Sul.

\section{RESULTADOS}

\section{Características gerais da microfauna}

A microfauna de foraminíferos planctônicos do intervalo estudado na sondagem 1-SCS-3B é representada por 60 espécies e subespécies, distribuídas em 13 gêneros (Anexo 1), totalizando 2.026 indivíduos, sendo que destes 209 exemplares foram identificados apenas em nível genérico (Globigerina sp.).

De um modo geral, a microfauna de foraminíferos planctônicos é pobre predominam os espécimes dos gêneros Globigerinoides e Globigerina, os quais correspondem a $36,80 \%$ e $36,68 \%$, respectivamente, dos exemplares identificados (Figura 2). Este fato foi anteriormente constatado por Closs (1966b), que analisando poços perfurados na porção emersa da bacia, assinalou a ausência da maioria das espécies de Globorotalia e a predominância de espécies do gênero Globigerinoides na seção miocênica.

Em relação ao estado de preservação dos exemplares, do

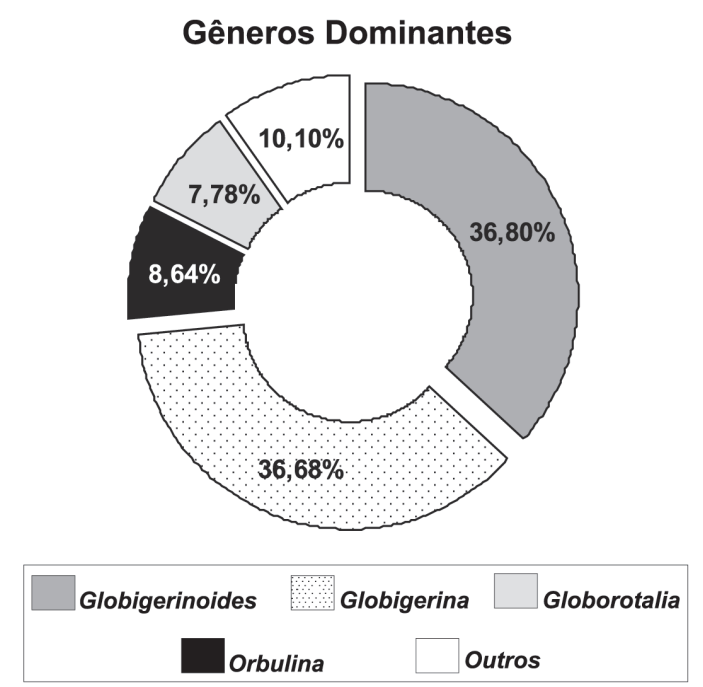

Figura 2. Gêneros dominantes de foraminíferos planctônicos do poço 1-SCS-3B.

Figure 2. Planktonic foraminifers dominant genera of the drill hole 1-SCS-3B. topo para a base até a profundidade de $615 \mathrm{~m}$, intercalam-se níveis de testas bem preservadas e níveis de testas oxidadas, que de modo geral apresentam um conteúdo fossilífero pobre. (Figura 3). Abaixo dos $615 \mathrm{~m}$ de profundidade aumenta o número de foraminíferos planctônicos e observa-se a ocorrência de níveis com carapaças em bom estado de preservação, porém, recobertas por um filme de óxido de ferro facilmente removível. Nas demais amostras abaixo de $870 \mathrm{~m}$ de profundidade os espécimes estão bem preservados, ainda que na sua maioria preenchidos por sedimentos recristalizados. por vezes exibem crescimentos de cristais de calcita.

É importante ressaltar as testas recobertas por óxido de ferro se encontram em sua grande maioria em excelente estado de preservação, tendo, portanto, a morfologia da parede em perfeito estado e que o sedimento de onde estas foram recuperadas também está oxidado. Não foram observados sinais de abrasão que indicariam que estas testas foram redepositadas de seqüências sedimentares mais antigas expostas e erodidas. Além disso, as espécies identificadas que apresentaram este tipo de preservação estão distribuídas de forma coerente dentro da sequiência bioestratigráfca, como será mostrado adiante ao se tratar da distribuição dos microfósseis, o que se constitui em mais um indício da sua condição de material in situ.

A atuação de processos diagenéticos na sucessão sedimentar estudada pode explicar a ocorrência destes intervalos oxidados. Uma hipótese que pode ser aventada e futuramente testada através de estudos paleoambientais com base em foraminíferos bentônicos, incluindo estudos de fóssildiagênese é o controle dos eventos de transgressão-regressão nestes processos diagenéticos.

Os eventos de transgressão-regressão controlam a posição e a dinâmica das zonas de mistura de águas intersticiais meteórica e marinha, promovendo diversas reações diagenéticas. Eventos regressivos podem expor extensas áreas da plataforma, aumentado a zona de recarga e, conseqüentemente, causando a incursão de águas meteóricas em direção à bacia. Associados este processo ocorrem dissolução de cimentos dolomíticos, formação de caolinita e óxidos e oxihidróxidos de ferro (Morad et al., 2000).

\section{Bioestratigrafia}

Um zoneamento bioestratigráfico é apresentado para a seção estudada do poço 1-SCS-3B, no qual foram reconhecidas oito das zonas propostas por Bolli \& Saunders (1985) para baixas latitudes. Assim sendo, o presente biozoneamento está baseado nos critérios empregados, para o mesmo intervalo de tempo, para definir as zonas de Bolli \& Saunders (1985) e por esta razão, equivale ao dos autores supracitados.

As zonas aqui estabelecidas são do tipo zona-de-intervalo, as quais são definidas por bio-horizontes distintos. Como conseqüência da utilização de amostras de calha foram tomados bio-horizontes referentes a níveis de desaparecimento de táxons particulares. As zonas assim definidas são comumente referidas como "zonas diferenciais superiores". 


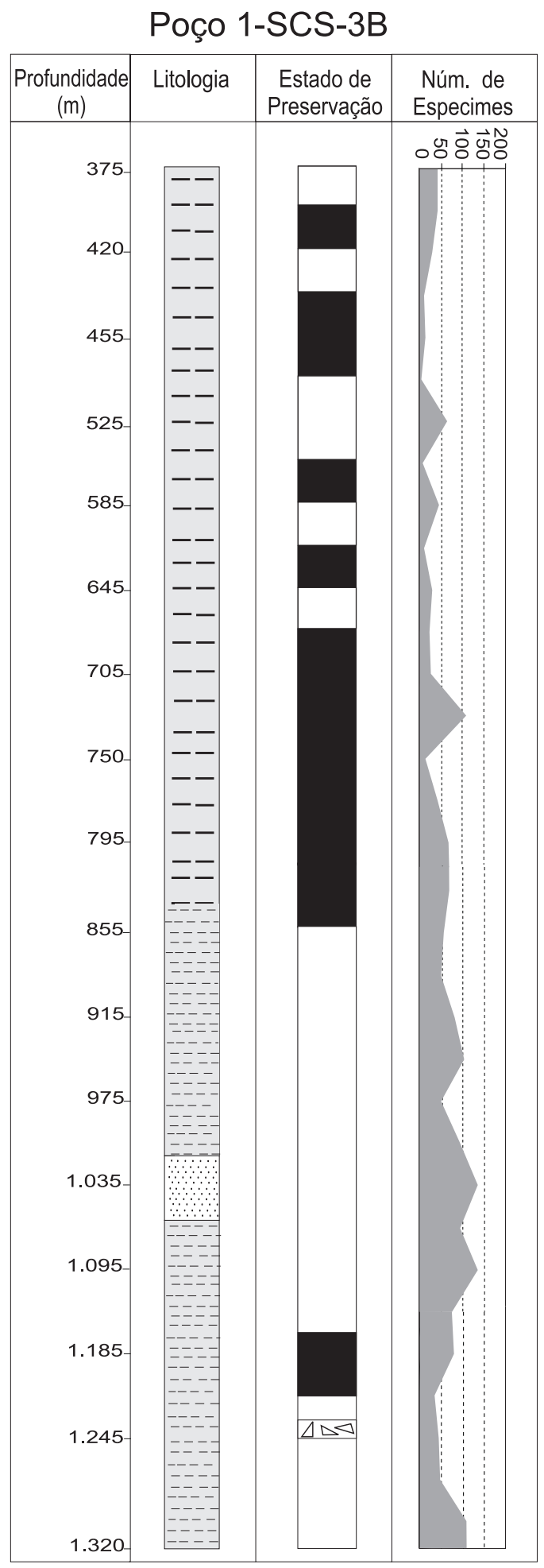

Convenções:

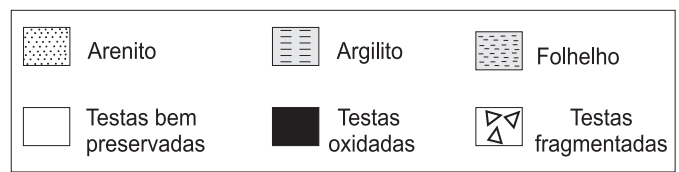

Figura 3. Características gerais da microfauna da sondagem 1SCS-3B.

Figura 3. General characteristics of the microfauna of the drill hole 1-SCS-3B.
De acordo com a distribuição das espécies e subespécies de foraminíferos planctônicos, foram reconhecidas as zonas de intervalo do tipo "diferencial superior", as quais designadas por ordem estratigráfica, são: Catapsydrax dissimilis, Catapsydrax stainforthi, Globorotalia fohsi fohsi, Globigerinoides ruber, Globorotalia. mayeri, Globorotalia acostaensis/Globorotalia menardii, Globorotalia margaritae evoluta e Globigerinoides trilobus fistulosus (Figura 4). O limite superior de cada biozona é dado pelo nível de extinção da(s) forma(s)-guia que caracteriza, na profundidade mais rasa da última amostra incluída, e a base é dada pelo nível de extinção da(s) espécie(s)-índice que caracteriza(m) a zona precedente. A Figura 5 ilustra os táxons que constituem as formas-guia utilizadas na identificação das biozonas supracitadas.

Zona: Catapsydrax dissimilis

Idade: Eomioceno.

Definição: Intervalo entre o último aparecimento de Globorotalia opima opima e o nível de extinção de Globigerina ciperoensis angustiumbilicata.

Ocorrência: Entre 1.260 e $1.320 \mathrm{~m}$ de profundidade.

Formas associadas: Globigerina euapertura, Globigerina venezuelana, Globigerina woodi, Globorotalia fohsi peripheroronda, Globorotalia fohsi fohsi, Globoquadrina altispira altispira, Globoquadrina dehicens, Globigerinoides ruber, Globigerinoides trilobus immaturus, Globigerinoides trilobus sacculifer, Globigerinoides trilobus trilobus, Globorotalia continuosa, Orbulina suturalis, Orbulina universa.

Zona: Catapsydrax stainforthi

Idade: Eomioceno.

Definição: Intervalo entre o último aparecimento de Globigerina ciperoensis angustiumbilicata e o nível de extinção de Catapsydrax dissimilis.

Ocorrência: Entre 1.260 e $1.230 \mathrm{~m}$ de profundidade.

Formas associadas: Globigerina druryi, Globigerina woodi, Globigerinoides ruber, Globigerinoides trilobus immaturus, Globigerinoides trilobus sacculifer, Globigerinoides trilobus trilobus, Globoquadrina altispira altispira, Globoquadrina dehicens, Globorotalia continuosa, Globorotalia fohsi peripheroacuta, Globorotalia fohsi peripheroronda, Globorotalia obesa, Globorotaloides variabilis, Orbulina suturalis, Orbulina universa.

Zona: Globorotalia fohsi fohsi

Idade: Mesomioceno.

Definição: Intervalo entre o último aparecimento de Catapsydrax dissimilis e a última ocorrência de Globorotalia fohsi peripheroacuta e Globorotalia fohsi peripheroronda. Ocorrência: Entre 1.200 e $1.230 \mathrm{~m}$ de profundidade.

Formas associadas: Globigerina druryi, Globigerina venezuelana, Globigerina woodi, Globigerinoides ruber, Globigerinoides trilobus sacculifer, Globigerinoides trilobus sacculifer, Globigerinoides trilobus trilobus, Globorotalia continuosa, Globorotalia mayeri, Globorotalia obe- 


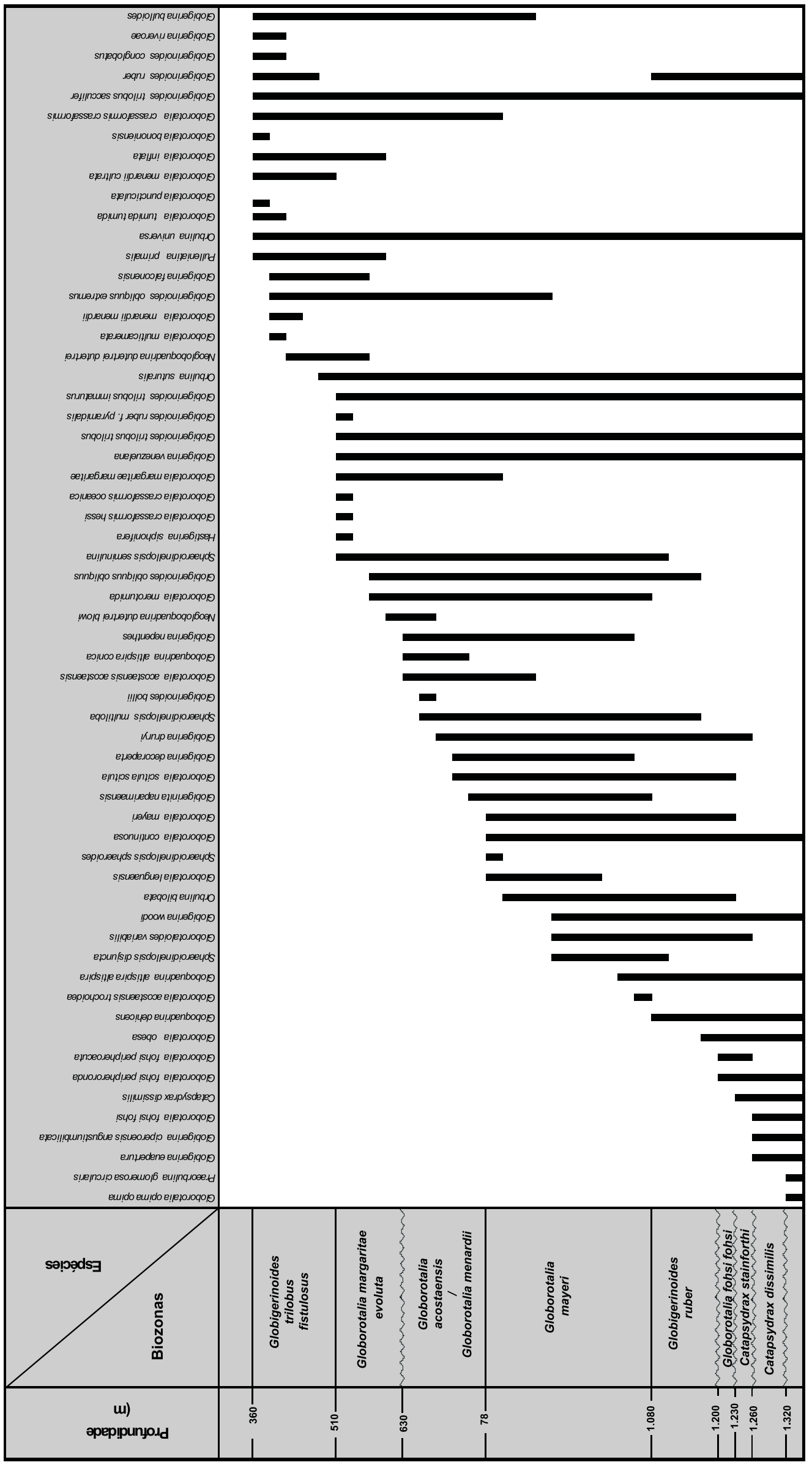


sa, Globorotalia scitula scitula, Globorotaloides variabilis, Globoquadrina altispira altispira, Globoquadrina dehicens, Orbulina bilobata, Orbulina suturalis, Orbulina universa.

Zona: Globigerinoides ruber

Definição: Intervalo entre o último aparecimento de Globorotalia fohsi peripheroacuta e Globorotalia fohsi peripheroronda e o nível da última ocorrência miocênica de Globigerinoides ruber.

Ocorrência: Entre 1.200 e $1.080 \mathrm{~m}$ de profundidade.

Comentário: Globoquadrina dehicens e Globorotalia obesa extinguem-se nesta zona.

Formas Associadas: Globigerina druryi, Globigerina venezuelana, Globigerina woodi, Globigerinoides obliquus obliquus, Globigerinoides trilobus immaturus, Globigerinoides trilobus sacculifer, Globigerinoides trilobus trilobus, Globorotalia continuosa, Globorotalia mayeri, Globorotalia scitula scitula, Globorotaloides variabilis, Globoquadrina altispira altispira, Orbulina bilobata, Orbulina suturalis, Orbulina universa, Sphaeroidinellopsis multiloba.

Zona: Globorotalia mayeri

Idade: Mesomioceno.

Definição: Intervalo entre a última ocorrência miocênica de Globigerinoides ruber e o último aparecimento de Globorotalia mayeri e Globorotalia continuosa.

Ocorrência: Entre 1.080 e $780 \mathrm{~m}$ de profundidade

Comentário: Extinguem-se nesta biozona Globorotalia merotumida, Globorotaloides variabilis e Globoquadrina altispira altispira.

Formas associadas: Globigerina bulloides, Globigerina decoraperta, Globigerina druryi, Globigerina nepenthes, Globigerina venezuelana, Globigerinita naparimaensis, Globigerinoides obliquus extremus, Globigerinoides obliquus obliquus, Globigerinoides trilobus immaturus, Globigerinoides trilobus sacculifer, Globigerinoides trilobus trilobus, Globorotalia acostaensis acostaensis, Globorotalia crassaformis crassaformis, Globorotalia mayeri, Globorotalia margaritae margaritae, Globorotalia scitula scitula, Orbulina bilobata, Orbulina suturalis, Orbulina universa, Sphaeroidinellopsis multiloba, Sphaeroidinellopsis seminulina.

Zona: Globorotalia menardii / Globorotalia acostaensis Idade: Mesomioceno/ Neomioceno.

Definição: Intervalo entre o nível de extinção de Globorotalia mayeri e o último aparecimento de Sphaeroidinellopsis multiloba.

Ocorrência: Entre 780 e $630 \mathrm{~m}$ de profundidade.

Comentário: Não foi possível individualizar as biozonas Globorotalia menardii e Globorotalia acostaensis. Optouse por representar uma biozona composta, pois a utilização de amostras de calha impossibilita a determinação do primeiro aparecimento de Globorotalia acostaensis, critério utilizado na definição do topo da zona de Globorotalia menardii e base da zona de Globorotalia acostaensis.

O topo da zona composta corresponde ao último aparecimento de Sphaeroidinellopsis multiloba, a qual, de acordo com alcance estratigráfico apresentado por Bolli \& Saunders (1985) definiria o topo da zona Globorotalia acostaensis; a base equivale ao nível de extinção de Globorotalia mayeri. Nesta biozona também se extinguem Globigerina druryi, Globigerina decoraperta, Globorotalia scitula scitula e Globigerinita naparimaensis.

Formas associadas: Globigerina bulloides, Globigerina venezuelana, Globigerinoides obliquus extremus, Globigerinoides obliquus obliquus, Globigerinoides trilobus immaturus, Globigerinoides trilobus sacculifer, Globigerinoides trilobus trilobus, Globorotalia crassaformis crassaformis, Globorotalia margaritae margaritae, Neogloboquadrina dutertrei blowi, Orbulina suturalis, Orbulina universa, Sphaeroidinellopsis seminulina.

Subzona: Globorotalia margaritae evoluta

Idade: Plioceno inferior (sensu Bergreen et al., 1995).

Definição: Intervalo entre o último aparecimento de Sphaeroidinellopsis multiloba e o nível de extinção de Globorotalia margaritae margaritae.

Ocorrência: Entre 630 e $510 \mathrm{~m}$ de profundidade.

Comentário: Extinguem-se na zona Globigerina venezuelana, Globigerina nepenthes, Globigerinoides obliquus obliquus, Globoquadrina altispira conica, Globorotalia acostaensis e Neogloboquadrina dutertrei blowi.

Formas associadas: Globigerina bulloides, Globigerina falconensis, Globigerinoides obliquus extremus, Globigerinoides trilobus sacculifer, Globorotalia crassaformis crassaformis, Globorotalia inflata, Neogloboquadrina dutertrei dutertrei, Orbulina suturalis, Orbulina universa, Pulleniatina primalis.

Subzona: Globigerinoides trilobus fistulosus Idade: Plioceno inferior (sensu Bergreen et al., 1995).

Definição: Intervalo entre o nível de extinção de Globorotalia margaritae margaritae e o último aparecimento de Globorotalia bononiensis.

Ocorrência: Entre 510 e 360m de profundidade.

Comentário: O nível de extinção de Globorotalia bononiensis indica o topo da zona de Globigerinoides trilobus fistulosus. Como não ocorrem outras espécies diagnósticas e não há amostras mais rasas na seção analisada, não é possível assegurar que o topo desta zona corresponda à referida profundidade. Neste intervalo extinguem-se Globigerinoides obliquus extremus, Neogloboquadrina dutertrei dutertrei e Globigerina falconensis.

Formas associadas: Globigerina bulloides, Globigerina riveroae, Globigerinoides conglobatus, Globigerinoides ruber, Globigerinoides trilobus sacculifer, Globorotalia bononiensis, Globorotalia inflata, Globorotalia menardii cultrata, Globorotalia puncticulata, Globorotalia tumida tumida, Orbulina universa, Pulleniatina primalis.

\section{DISCUSSÃO}

Com base na distribuição da microfauna de foraminíferos planctônicos, foram reconhecidas oito biozonas para a se- 


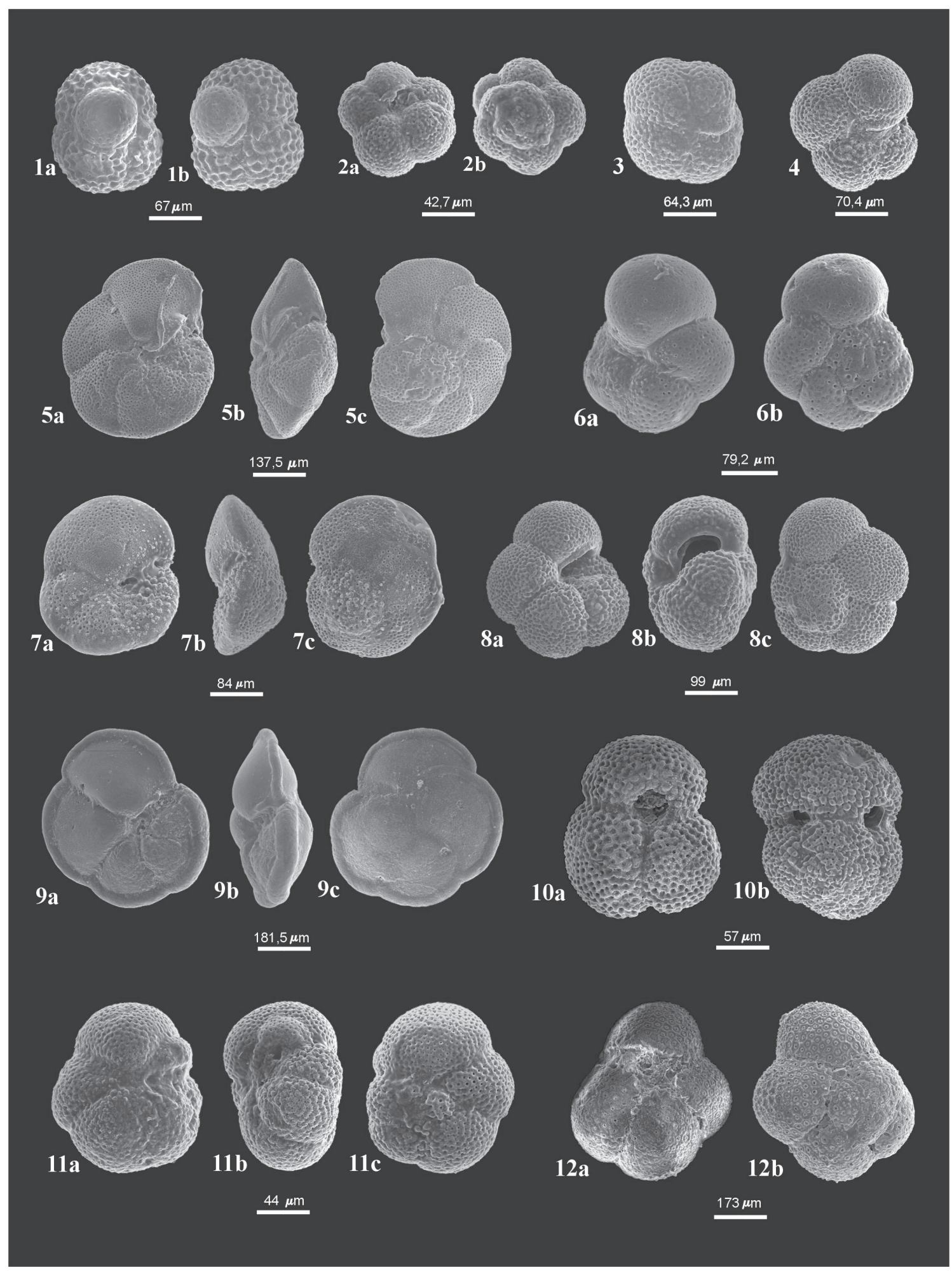

Figura 5. 1. Catapsydrax dissimilis, MP-F-3085, a - vista umbilical (umbilical view), b-vista lateral (lateral view); 2. Globigerina ciperoensis angustiumbilicata, MP-F-3086, a - vista umbilical (umbilical view), b - vista espiral (spiral view); 3. Globorotalia acostaensis, MP-F-3087, vista umbilical (umbilical view); 4. Globorotalia continuosa, MP-F-3088, vista umbilical (umbilical view); 5. Globorotalia fohsi peripheroacuta, MP-F-3089, a - vista umbilical (umbilical view), b - vista lateral (lateral view), c - vista espiral (spiral view); 6. Globorotalia fohsi peripheroronda, MP-F-3090, a - vista umbilical (umbilical view), b -vista espiral (spiral view); 7. Globorotalia margaritae margaritae, MPF-3091, a - vista umbilical (umbilical view), b - vista lateral (lateral view), c - vista espiral (spiral view); 8. Globorotalia mayeri, MP-F-3092, a - vista umbilical (umbilical view), b - vista lateral (lateral view), c - vista espiral (spiral view); 9. Globorotalia menardii menardii, MPF-3093, a - vista umbilical (umbilical view), b - vista lateral (lateral view), c - vista espiral (spiral view); 10. Globigerinoides ruber, MPF-3094, a - vista umbilical (umbilical view), b - vista lateral (lateral view); 11. Globorotalia opima opima, MP-F-3095,a - vista umbilical (umbilical view), b - vista lateral (lateral view), c - vista espiral (spiral view); 12. Sphaeroidinellopsis multiloba, MP-F-3096, a - vista umbilical (umbilical view), b - vista espiral (spiral view). 
Mioceno e duas no Plioceno inferior. As seções correspondentes ao Plioceno superior e ao Quaternário não foram amostradas.

A sondagem 1-SCS-3B foi anteriormente estudada por Koutsoukos (1982) e Gomide (1989), os quais propuseram biozonas baseadas na análise de foraminíferos planctônicos e nanofósseis calcários, respectivamente.

Koutsoukos (1982) reconheceu oito biozonas informais utilizadas pela Petrobrás na seção neogênica do poço 1SCS-3B que correspondem a dez biozonas sensu Blow (1969), sendo oito para o Mioceno e duas para o Plioceno/Quaternário. Gomide (1989) identificou nove biozonas para o Neogeno do mesmo poço, sendo seis para o Mioceno e duas para o Plioceno inferior. Uma comparação entre o esquema zonal aqui proposto e aqueles estabelecidos por Gomide (1989) e Koutsoukos (1982) para a sondagem é apresentada na Figura 6. Esta comparação mostra diversas discrepâncias, as quais podem ser atribuídas à utilização de amostras de calha em ambos os trabalhos, que possuem baixa resolução bioestratigráfica, e a diferentes malhas de amostragem adotadas. Vale ressaltar que o esquema zonal apresentado por Koutsoukos (1982) foi baseado nos biozoneamentos propostos por Bolli (1957), Noguti \& Santos (1972), Noguti (1975) e Blow (1969), o que, em suma, implica na utilização de outras espécies no estabelecimento das biozonas, bem como numa mescla de critérios.

Tanto no esquema zonal aqui proposto quanto naquele estabelecido por Koutsoukos (1982), o Mioceno inferior está representado apenas pela sua porção inferior. Entretanto, reconhecemos o limite entre o Mioceno inferior e o Oligoceno como um hiato que separa a Zona de Catapsydrax dissimilis e a Zona de Globorotalia opima opima (Figura 6), a qual foi caracterizada pela ausência da zona de Globigerina ciperoensis (sensu Bolli \& Saunders, 1985), enquanto que o autor supracitado não considera tal hiato. Em contrapartida, Gomide (1989) reconheceu o Mioceno inferior completo, tendo identificado três biozonas neste intervalo (N-570 Helicosphaera ampliaperta; N-560 Sphenolithus heteromorphus e N-550 Triquetrohabdulus carinatus).

Nos biozoneamentos apresentados neste trabalho e em Koutsoukos (1982), um hiato separa o Mioceno inferior do Mioceno médio, tendo esta sido posicionada a profundidades bastante próximas (1.230m e $1.244 \mathrm{~m}$, respectivamente). Embora não apresentando um hiato associado, o limite entre o Mioceno inferior e médio estabelecido por Gomide (1989) está mais próximo (posicionado a aproximadamente $1.220 \mathrm{~m}$ ) em relação ao estabelecido neste trabalho.

Koutsoukos (1982) propôs uma discordância entre o Mioceno médio e o Mioceno superior apesar de haver correlacionado seu biozoneamento com sucessivas zonas de Blow (1969) sem que haja a ausência de alguma biozona deste no mesmo intervalo. No presente trabalho, assim como no de Gomide (1989), a presença deste hiato não foi evidenciada. Contudo, no esquema zonal aqui proposto estes períodos não foram individualizados, já que não foi possível separar as biozonas Globorotalia acostaensis e Globorotalia menardii que constituem uma biozona composta (Figura 6).
O limite entre o Mioceno médio/superior e o Plioceno foi caracterizado como um hiato, devido à ausência da zona de Globorotalia humerosa e da base da zona Globorotalia margaritae, representada pela subzona Globorotalia margaritae margaritae (sensu Bolli \& Saunders, 1985). O Plioceno inferior tardio, ou a parte superior do Plioceno inferior (sensu Berggren, 1995), está representado apenas pela subzona Globigerinoides trilobus fistulosus (Figura 6), que corresponde à base da zona de Globorotalia miocenica (sensu Bolli \& Saunders, 1985). Entretanto, como não havia amostras mais rasas disponíveis, não se pode afirmar a exata profundidade do topo desta zona, já que o nível de extinção de Globorotalia bononiensis pode estar mais acima na seção.

A comparação entre a cronoestratigrafia proposta neste trabalho e aquelas estabelecidas por Koutsoukos (1982) e Gomide (1989) para a sondagem em questão (Figura 6), evidencia que o esquema biostratigráfico aqui estabelecido implica na redução da seção sedimentar neogênica na sondagem 1-SCS-3B.

\section{CONCLUSÕES}

A análise da distribuição das espécies de foraminíferos planctônicos ao longo do poço 1 -SCS-3B revelou uma microfauna pobre, porém, bastante diversificada, o que possibilitou o posicionamento da seção neogênica da referida sondagem entre 1.320 e $360 \mathrm{~m}$ de profundidade.

Os critérios para o reconhecimento das diferentes biozonas que constituem o esquema padrão estabelecido para mares tropicais e temperados de Bolli \& Saunders (1985) foram empregados, tendo sido reconhecidas seis zonas e duas subzonas que caracterizam este esquema e, portanto, evidenciam ao menos quatro hiatos importantes situados no limite Mioceno-Oligoceno, no Mioceno inferior, no limite Mioceno inferior-Mioceno médio e aquele que marca o limite Mioceno-Plioceno. Destes, apenas o hiato situado no Mioceno inferior-médio havia sido documentado previamente por Koutsoukos (1982).

De acordo com as informações disponíveis, não é possível inferir a natureza destes hiatos, porém se levarmos em conta os trabalhos realizados na porção emersa da bacia (Sanguinetti, 1979, 1980; Ornellas, 1981; Thiesen \& Madeira-Falcetta, 1984; Madeira-Falcetta \& Thiesen (1988), Carreño et al., 1997, 1999;), podemos hipotetizar uma possível influência de eventos transgressivos associados. Análises de foraminíferos bentônicos poderão testar esta hipótese.

De modo semelhante, a análise da distribuição de foraminíferos planctônicos presentes ao longo de outros poços efetuados na porção offshore permitirá estabelecer, com um maior grau de confiança a aplicação do esquema zonal de Bolli \& Saunders (1985) e, portanto, propor a sua extensão para toda a bacia.

Além dos dados aqui apresentados, a análise atualmente em curso de amostras mais profundas na seção em apreço tem revelado a ocorrência de espécies do Oligoceno inferior coexistindo 


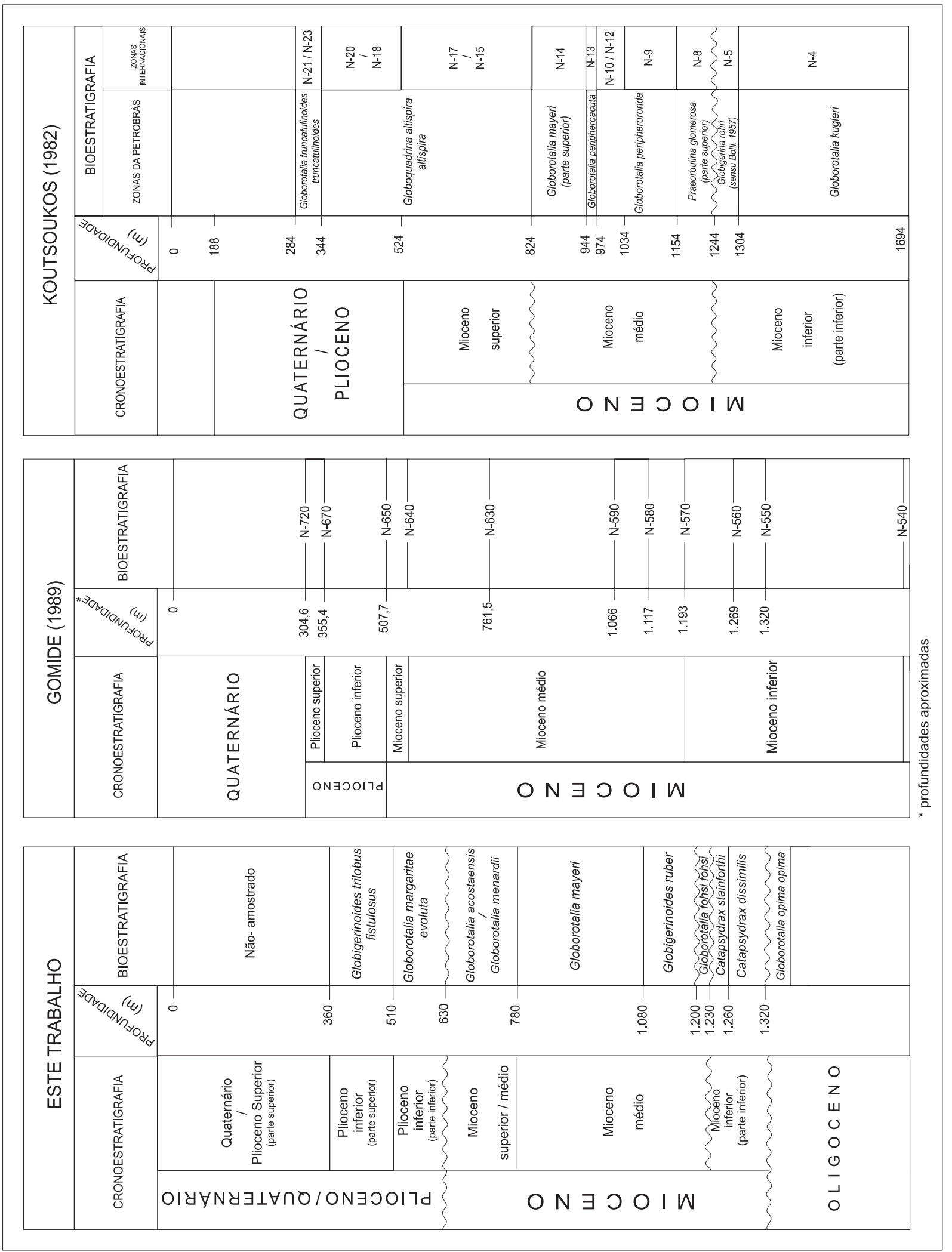

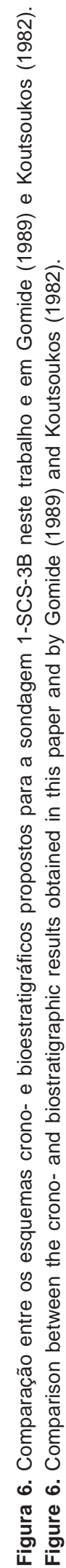


com as mesmas espécies identificadas na seção neogênica, estas últimas seguramente ocorrendo devido à contaminação por desmoronamento da parede do poço. Também vale ressaltar que do ponto de vista do estado de preservação ocorre uma brusca mudança na seção subjacente ao intervalo estudado, onde os espécimes se encontram em sua maioria recristalizados.

\section{AGRADECIMENTOS}

As autoras agradecem à Universidade Federal do Rio Grande do Sul pelo suporte do Programa de Pós-Graduação em Geociências e do Centro de Microscopia Eletrônica. A João Carlos Coimbra (UFRGS) e a Henrique Zerfass (CPRM) pela leitura crítica do manuscrito. A Luiz Fernando De Ros (UFRGS) pela sugestão de bibliografia e discussões acerca de processos diagenéticos. Aos revisores por suas excelentes sugestões e enriquecedores questionamentos. G. S. Anjos agradece à Agência Nacional do Petróleo (ANP) pela concessão da bolsa de estudos.

\section{REFERÊNCIAS}

Asmus, H.E. 1983. A Bacia de Pelotas no Esquema Evolutivo da Margem Continental Brasileira. In: SIMPÓSIO SUL-BRASILEIRO DE GEOLOGIA, 1, 1983. Atas, Porto Alegre, SBG, p. 245-252.

Berggren, W.A., Hilgen, F.J., Langereis, C.G., Kent, D.V., Obradovich, J.D., Raffi, I., Raymo, M.E. \& Shackleton, N.J. 1995. Late Neogene chronology: new perspectives in highresolution stratigraphy. Geological Society American Bulletin, 107: 1272-1287.

Bertels, A. \& Madeira-Falcetta, M. 1977. Neogene Foraminifera of the South Atlantic. In: F.M. Swain (ed.) Stratigraphic Micropaleontology of Atlantic Basins and Borderlands, Elsevier, p. $441-465$.

Blow, W. 1969. Late middle Eocene to Recent planktonic foraminiferal biostratigraphy. In: R. Brönnimann, \& H.H. Renz (eds.) INTERNATIONAL CONFERENCE ON PLANKTONIC MICROFOSSILS, 1, 1967. Proceedings, Genebra, p. 199-422.

Bolli, H.M. 1957. Planktonic foraminifera form the OligoceneMiocene Cipero and Lengua Formations of Trinidad, B.W.I. United States National Museum Bulletin, 215:97123.

Bolli. H.M. \& Premoli Silva, I. 1973. Oligocene to Recent planktonic foraminifera and stratigraphy of the Leg 15 Sites in the Caribbean Sea. Initial Report of the Deep Sea Drilling Project, 15:475-497.

Bolli, H.M. \& Saunders, J.B. 1981. The species of Sphaeroidinellopsis Banner \& Blow, 1959. Cahiers de Micropaléontologie, 4: 13-24.

Bolli, H.M. \& Saunders, J.B. 1982. Globorotalia mayeri and its relationship to Globorotalia siakensis and Globorotalia continuosa. Journal of Foraminiferal Research, 12:39-50.

Bolli, H.M. \& Saunders, J.B. 1985. Oligocene to Holocene low latitude planktic foraminifera. In: H.M. Bolli; J.B. Saunders \& K. Perch-Nielsen (eds.) Plankton Stratigraphy, Cambridge University Press, p. 155-262.

Boltovskoy, E.; Thiesen, Z.V. \& Falcetta, M.M. 1983. Foraminíferos de cuatro perforaciones de la planicie de Rio Grande do Sul (Brasil). Pesquisas, 15:127-149.
Carmo, D.A. \& Sanguinetti, Y. 1999. Taxonomy and paleocenographical significance of the genus Krithe (Ostracoda) in the Brazilian margin. Journal of Micropalenontology, 18:111-123.

Carreño, A.L.; Coimbra, J.C. \& Sanguinetti, Y.T. 1997. Biostratigraphy of the Late Neogene and Quaternary ostracodes in the Pelotas Basin, southern Brazil. Gaia, 14:33-43.

Carreño, A.L.; Coimbra, J.C. \& Carmo, D.A. 1999. Late Cenozoic sea level changes evidenced by ostracodes in the Pelotas Basin, southernmost Brazil. Marine Micropaleontology, 37:117-129.

Closs, D. 1966a. The presence and stratigraphical importance of the Orbulina surface in southern Brazil. Notas e Estudos (Escola de Geologia de Porto Alegre), 1(2):3-8.

Closs, D. 1966b. Cenozoic stratigraphy of the southern Brazil. In: WEST AFRICAN MICROPALEONTOLOGICAL COLLOQUIUM, 2, 1965. Proceedings, Ibadan, p. 34-44.

Closs, D. 1967. Miocene planktonic foraminifera from southern Brazil. Micropaleontology, 13(3):337-344.

Closs, D. \& Madeira, M.L. 1968. Cenozoic Foraminífera from the Chuy Drill Hole, Northern Uruguay. Ameghiniana, 5(7):229 -246

Closs, D. 1970. Estratigrafia da Bacia de Pelotas, Rio Grande do Sul. Iheringia (Série Geologia), 3:3-37.

Daemon, R.F. 1969. Estudo bioestratigráfico preliminar efetuado em sedimentos neogênicos dos poços 2-PS-1-RS (Palmares do Sul, RS) 2-MO-1-RS (Mostardas, RS) e 2-CI-1 (Cassino, RS). Relatório 390, Petrobrás, Distrito de Exploração do Sul, 17 p. (inédito).

Dias, J.L.; Sad, A.R.E.; Fontana, R.L. \& Feijó, F.J. 1994. Bacia de Pelotas. Boletim de Geociências da Petrobrás, 8(1):235-245.

Ellis, B.F. \& Messina, A. 1995. Catalogue of Foraminifera in CD ROM. American Museum of Natural History, Nova Iorque.

Fernandes, J.M.G. 1975. O Gênero Uvigerina (Foraminiferida) do Cenozóico Superior da Bacia de Pelotas. Programa de Pósgraduação em Geociências, Universidade Federal do Rio Grande do Sul, Dissertação de Mestrado, 122 p.

Fontana, R. L. 1996. Geotectônica e sismoestratigrafia da Bacia de Pelotas, Plataforma de Florianópolis. Programa de Pós-Graduação em Geociências, Universidade Federal do Rio Grande do Sul, Tese de Doutorado, 214 p.

Gamboa, L.A.P.\& Rabinovitch, P.D. 1981. The Rio Grande fracture zone in the western South Atlantic and its tectonics implications. Earth and Planetary Sciences Letters, 52:410-418.

Gomide, J. 1989. Bacia de Pelotas - Biocronoestratigrafia baseada em nanofósseis calcários. In: CONGRESSO BRASILEIRO DE PALEONTOLOGIA, 11, 1989. Resumos expandidos, Curitiba, SBP, p.338-351.

Gonçalves, A.; Oliveira, M.A.M. \& Mota, S.O.M. 1979. Geologia da Bacia de Pelotas e da Plataforma de Florianópolis. Boletim Técnico da Petrobrás, 22(3):155-226.

Kotzian, S.B. \& Eilert, V. 1985. Ostracodes Mio-pleistocênicos da perfuração Chuí no364 (Bacia de Pelotas), Uruguai. Acta Geológica Leopoldensia, 9(29):81-94.

Kennet, J.P. \& Srinivasan, S. 1983. Neogene planktonic foraminifera: a phylogenetic atlas. $1^{\mathrm{a}}$ ed. Stroudsburg, Hutchinson Ross Publishing Company, 265p.

Koutsoukos, E.A.M. 1982. Geohistória e Paleoecologia das Bacias Marginais de Florianópolis e Santos. In: CONGRESSO BRASILEIRO DE GEOLOGIA, 32, 1982. Resumos expandidos, Salvador, SBG, 5:2369-2382.

Loeblich, A.R. \& Tappan, H. 1988. Foraminiferal genera and their classification. $1^{\mathrm{a}}$ ed. Nova Iorque, Van Nostrand Reinhold Company, 2 vol., 970 p. +847 p.

Madeira-Falcetta, M. \& Thiesen, Z.V. 1988. Foraminíferos da perfuração 2-GAst-1A-RS, Bacia de Pelotas. Pesquisas, 21: 49-59. 
Madeira-Falcetta, M.; Thiesen, Z.V.; Kotzian, S.B. \& Godolphim, M. 1980. Interpretação de dois testemunhos de sondagem (PSD3 e ALB) na planície costeira do Rio Grande do Sul -Brasil. Pesquisas, 13:91-107.

Morad, S.; Ketzer, J.M. \& De Ros, L.F. 2000. Spatial and temporal distribution of diagenetic alterations in siliciclastics rocks: implications for mass transfer in sedimentary basins. Sedimentology, 47:95-120.

Noguti, I. 1975. Zonación Bioestratigrafica de los Foraminíferos Planctónicos del Terciário de Brasil. Revista Española de Micropaleontología, 7(3):391-401.

Noguti, I. \& Santos, J.F.S. 1972. Zoneamento Preliminar por Foraminíferos Planctônicos do Aptiano ao Mioceno na Plataforma Continental do Brasil. Boletim Técnico da Petrobrás, 15(3):265-283.

Ornellas, L.P. 1981. Os ostracodes e seu significado na interpretação dos eventos cenozóicos na Bacia de Pelotas, RS - transgressões, regressões e bioestratigrafia. Programa de Pós-graduação em Geociências, Universidade Federal do Rio Grande do Sul, Tese de Doutorado, $217 \mathrm{p}$.

Sanguinetti, Y. 1979. Miocene ostracodes of the Pelotas Basin, State of Rio Grande do Sul, Brazil. Pesquisas, 12:119-187.

Sanguinetti, Y. 1980. Bioestratigrafia (Ostracodes) do Mioceno da Bacia de Pelotas, Rio Grande do Sul. Pesquisas, 13:7-34.
Sanguinetti, Y; Ornellas, L.P. \& Coimbra, J.C. 1991. Post Miocene Ostracodes from Pelotas Basin, southern Brazil. Taxonomy Part I. Pesquisas, 18(2):138-155.

Sanguinetti, Y; Ornellas, L.P.; Coimbra, J.C. \& Ramos, M.I.F. 1992 Post Miocene Ostracodes from Pelotas Basin, southern Brazil. Taxonomy - Part II. Pesquisas, 19(2):155-166.

Sprechmann, P. 1978. The Paleoecology and Paleooceanography of the Uruguayan coastal area during the Neogene and Quaternary. Zitteliana, 4:3-72.

Stainforth, R.M.; Luterbacher, H.; Beard, J.H. \& Jeffords, J.M 1975. Cenozoic Planktonic Foraminiferal Zonation and Characteristics of Index Forms. University of Kansas, Paleontogical Contributions, Article 62:425 p.

Thiesen, Z.V. 1975. Bolivinitidae e Caucasinidae (Foraminiferida) do Cenozóico Superior da Bacia de Pelotas, Rio Grande do Sul. Acta Geológica Leopoldensia, 2(3):3-82.

Thiesen, Z.V. \& Madeira-Falcetta, M. 1984. Foraminíferos e a evolução paleoecológica da porção norte da província costeira do Rio Grande do Sul. In: CONGRESSO BRASILEIRO DE GEOLOGIA, 33, 1984. Resumos expandidos, Rio de Janeiro, SBG, p. 316-321. 
Anexo 1. Espécies de foraminíferos planctônicos identificadas.

Catapsydrax dissimilis Cushman \& Bermúdez, 1937

Globigerina bulloides d'Orbigny, 1826

Globigerina ciperoensis angustiumbilicata Bolli, 1957

Globigerina decoraperta Takayangi \& Saito, 1962

Globigerina druryi Akers, 1955

Globigerina euapertura Jenkins, 1960

Globigerina falconensis Blow, 1959

Globigerina nepenthes Todd, 1957

Globigerina riveroae Bolli \& Bermúdez, 1965

Globigerina venezuelana Hedberg, 1937

Globigerina woodi Jenkins, 1960

Globigerinita naparimaensis Brönnimann, 1951

Globigerinoides bollii Blow, 1959

Globigerinoides conglobatus Brady, 1879

Globigerinoides obliquus extremus Bolli \& Bermúdez, 1965

Globigerinoides obliquus obliquus Bolli, 1957

Globigerinoides ruber d'Orbigny, 1839

Globigerinoides ruber d'Orbigny, 1839 f. pyramidalis van den Broeck, 1876

Globigerinoides trilobus immaturus LeRoy, 1939

Globigerinoides trilobus sacculifer Brady, 1877

Globigerinoides trilobus trilobus Reuss, 1850

Globoquadrina altispira altispira Cushman \& Jarvis, 1936

Globoquadrina altispira conica Brönnimann \& Resig, 1971

Globoquadrina dehicens Chapman, Parr \& Collins, 1934

Globorotalia acostaensis acostaensis Blow, 1959

Globorotalia acostaensis trochoidea Bizon \& Bizon, 1965

Globorotalia bononiensis Dondi, 1963

Globorotalia continuosa Blow, 1959

Globorotalia crassaformis crassaformis Galloway \& Wissler, 1927

Globorotalia crassaformis hessi Bolli \& Premoli Silva, 1973

Globorotalia crassaformis oceanica Cushman \& Bermúdez, 1949

Globorotalia fohsi fohsi Cushman \& Ellisor, 1939

Globorotalia fohsi peripheroacuta Blow \& Banner, 1966

Globorotalia fohsi peripheroronda Blow \& Banner, 1966

Globorotalia inflata d'Orbigny, 1839

Globorotalia lenguaensis Bolli, 1957

Globorotalia margaritae margaritae Bolli \& Bermúdez, 1965

Globorotalia mayeri Cushman \& Ellisor, 1939

Globorotalia menardii cultrata d'Orbigny, 1839

Globorotalia menardii menardii Parker, Jones \& Brady, 1865

Globorotalia merotumida Blow \& Banner, 1965

Globorotalia multicamerata Cushman \& Jarvis, 1930

Globorotalia obesa Bolli, 1957

Globorotalia opima opima Bolli, 1957

Globorotalia puncticulata Deshayes, 1832

Globorotalia scitula scitula Brady, 1882

Globorotalia tumida tumida Brady, 1877

Globorotaloides variabilis Bolli, 1957

Hastigerina siphonifera d'Orbigny, 1839

Neogloboquadrina dutertrei blowi Rögel \& Bolli, 1973

Neogloboquadrina dutertrei dutertrei d'Orbigny, 1839

Orbulina bilobata d'Orbigny, 1846

Orbulina suturalis Brönnimann, 1951

Orbulina universa d'Orbigny, 1839

Praeorbulina glomerosa circularis Blow, 1956

Pulleniatina primalis Banner \& Blow, 1967

Sphaeroidinellopsis disjuncta Finlay, 1940

Sphaeroidinellopsis multiloba LeRoy, 1944

Sphaeroidinellopsis seminulina Schwager, 1866

Sphaeroidinellopsis sphaeroides Lamb, 1969 